\title{
CORRECTION
}

\section{Correction to: Adenovirus-mediated LIGHT gene modification in murine B-cell lymphoma elicits a potent antitumor effect}

Guili Hu' ${ }^{1}$ Yang $\mathrm{Liu}^{1}$, Hong $\mathrm{Li}^{2}$, Dekuang Zhao ${ }^{1}$, Liuqing Yang ${ }^{1}$, Jiangen Shen ${ }^{1}$, Xuejun Hong ${ }^{1}$, Xuetao Cao ${ }^{1,3}$ and Qingqing Wang ${ }^{1}$

Cellular \& Molecular Immunology (2020) 17:305-306; https://doi.org/10.1038/s41423-019-0356-4

Correction to: Cellular \& Molecular Immunology https://doi.org/ 10.1038/cmi.2010.15, published 26 April 2010

In this article, published online on 26 April 2010, there was an unintended error in Fig. 2a during proof revision process when submitted higher resolution figures as the typeset company needs, leading to that ICAM-1 FACS staining pictures are the same for A20/LacZ group and A20/LIGHT group. The picture of ICAM-1 FACS staining for A20/LacZ group has now been replaced with the correct picture which was shown in the proof. The corrected Fig. $2 a$ is shown here. We are sorry for the inconvenience caused by this error.

\footnotetext{
Institute of Immunology, Zhejiang University, Hangzhou, China; ${ }^{2}$ Molecular and Cellular Biology Group, Laboratory of Respiratory Biology, NIEHS/NIH, Research Triangle Park, NC, USA and ${ }^{3}$ National Key Laboratory of Medical Immunology \& Institute of Immunology, Second Military Medical University, Shanghai, China

Correspondence: Qingqing Wang (wqq@zju.edu.cn)

These authors contributed equally: Guili Hu, Yang Liu

Published online: 18 February 2020
} 
a
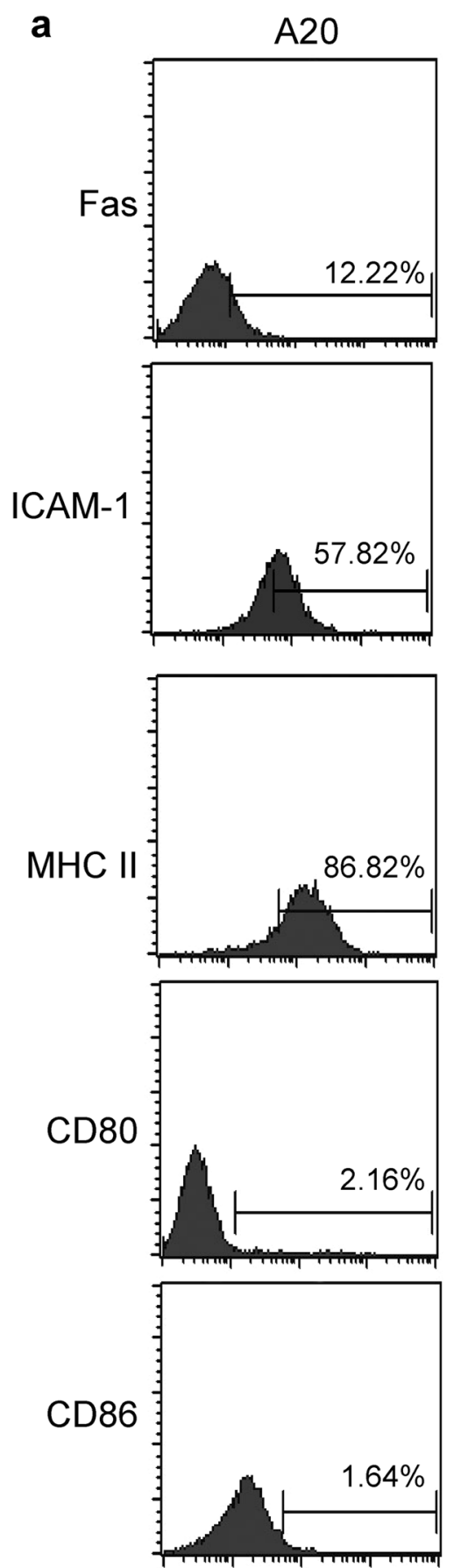
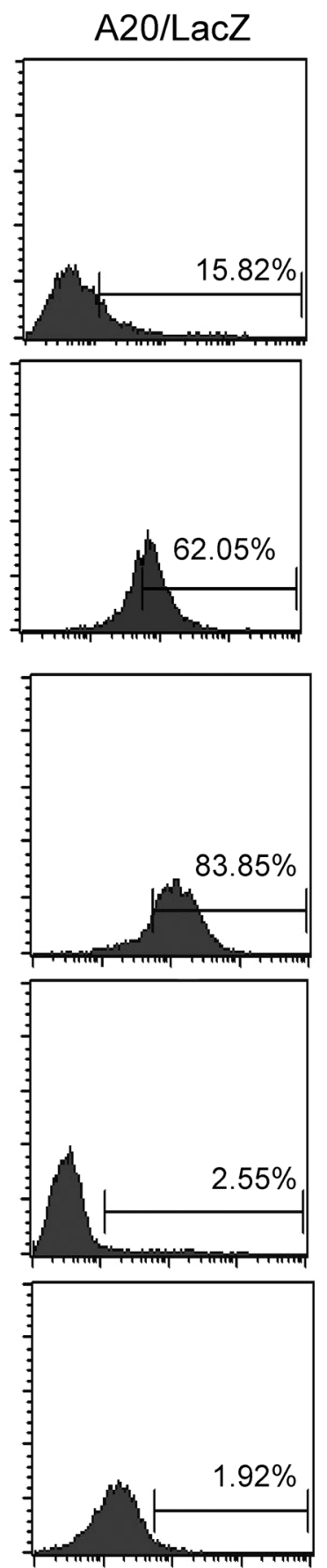
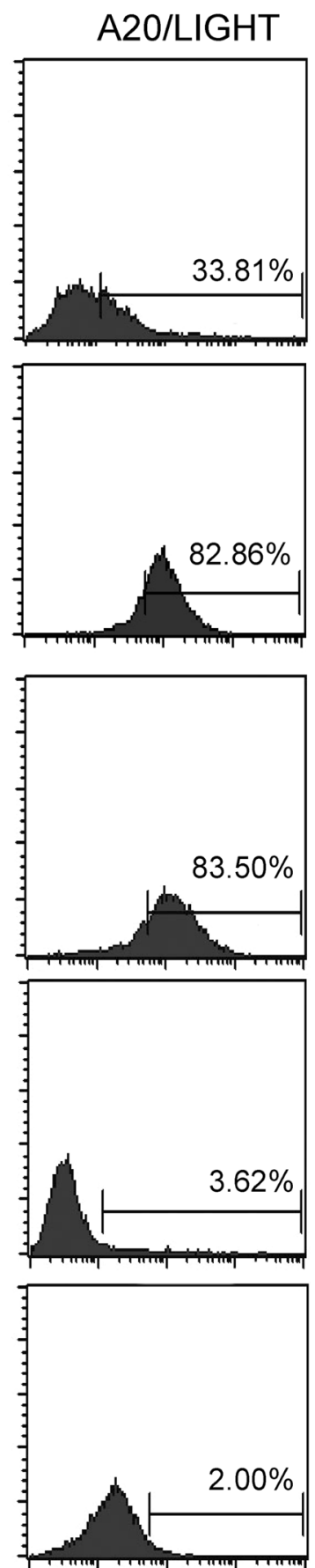

Fig. 2 